Rev. Adv. Mater. Sci. 56 (2018) 215-223

\title{
MONOLAYERS OF SURFACTANTS CONTAINING NANOPARTICLES OF HEXACYANOFERRATES OF VARIOUS METALS
}

\author{
N.A. Stepashkin, M.K. Chernenko, V.D. Khripun, N.S. Ivanov \\ and N.G. Sukhodolov
}

Institute of Chemistry, Saint Petersburg State University, Saint Petersburg, 198504, Russian Federation

Received: April 11, 2018

\begin{abstract}
In this article the possibility of obtaining Langmuir-Blodgett films on the basis of octadecylamine monolayers containing nanoparticles of hexacyanoferrates of various metals was shown. A comparison was made between different methods for obtaining these particles. Nanoparticles were produced using sodium hexametaphosphate as a stabilizer, their average size were determined by laser diffraction. The isotherms of compression were studied for monolayers on a subphase containing nanoparticles. Optimal conditions for the transfer of monolayers to a solid substrate were chosen according to the data obtained and their morphology was studied by scanning electron microscopy. The cyclic voltammetry method also confirmed the successful transfer of a monolayer to a solid substrate and showed the manifestation of the electrochemical properties of the obtained substrates.
\end{abstract}

\section{INTRODUCTION}

Prussian blue and other metals hexacyanoferrate $(\mathrm{HCF})$ with general formula $\mathrm{A}\left[\mathrm{B}(\mathrm{CN})_{6}\right] \times \mathrm{nH}_{2} \mathrm{O}$ (where $A$ and $B$ are atoms of transition metals) have been studied as promising compounds due to their magnetic properties [1], high ion-exchange capacity [2]. These hexacyanoferrates have shown the possibility of using electrochemical imaging devices $[3,4]$, catalysts [5], energy carriers [6], and ion-selective sensors $[7,8]$.

The preparation of nanoparticles can be carried out by synthesis in a microemulsion, by micellar synthesis in aqueous and non-aqueous solution, by a sol-gel method, by hydrolysis coprecipitation method.

The reagents are contained in small droplets that diffuse into each other over a very short period of time during the synthesis particles in a microemulsion. Microemulsions with large droplet sizes are thermodynamically unstable due to high surface tension at the water/oil boundary and tend to exfoliate. But it is possible to achieve droplet reduction to a value of the order of $5-50 \mathrm{~nm}$ and to obtain a stable microemulsion with certain amounts of surfactant and water/oil ratio. This value of droplet size corresponds to the size of very large micelles, so synthesis in microemulsion is similar to synthesis in micelles. Adding alcohols with an average length of the hydrocarbon chain as a cosolvent also contributes to the formation of a stable microemulsion. Mass transfer between the microemulsion phases can be carried out by increasing the electrolyte concentration (for ionic surfactants) or the temperature (for non-ionic surfactants).

There is a semi-empirical formula to estimate the average droplet size of a microimulsion:

$r_{\text {(hydrodynamic) }}=r_{\text {(core) }}+I_{\text {(tail) }}$

Corresponding author: N.A. Stepashkin, e-mail: stepashkin.nick17@gmail.com 
where $r_{\text {(hydrodynamic) }}$ is the hydrodynamic radius, $r_{\text {(core) }}$ is the droplet size, $I_{\text {(tail) }}$ is the length of the hydrocarbon chain of the surfactant. The droplet size is proportional to the length of the surfactant chain and the water/oil molar ratio $w_{0}$. We obtain, taking into account the assumption that the surfactant head groups are spherical and all surfactant molecules are located at the water/oil boundary:

$r_{\text {(core) }}=3 V_{m} w_{0} / a_{0}$

where $a_{0}$ is the area of the head group of the surfactant molecule, $V_{m}$ is the volume occupied by the hydrocarbon chain of the surfactant. The ratio $V_{m} / a_{0}$ is a constant and a unique characteristic of the surfactant.

Synthesis of nanoparticles is carried out by mixing two ideal microemulsions containing reagents, after which the surfactants are washed out of the formed precipitate. It is also possible to add an aqueous solution one of the reagents to the microemulsion containing the second reagent.

The disadvantages of the method include the difficulty in determining the empirical parameters specified above and, as a consequence, the difficulty of selecting surfactants, the water/oil ratio for the preparation of a microemulsion.

An approach using stabilizer substances is possible to obtain nanoparticles of complex salts, such as insoluble metal hexacyanoferrates,. Usually the salts of hexacyanoferrates are prepared by mixing aqueous solutions of metal salts and potassium $\mathrm{K}_{3}\left[\mathrm{Fe}(\mathrm{CN})_{6}\right]$ in a ratio of $3: 2$, which leads to the formation of insoluble precipitates. The reaction is carried out using sodium hexametaphosphate (SHMP) as a stabilizer to prevent precipitation and to form hexacyanoferrate nanoparticles. The presence of a cyclic polyphosphate containing six SHMP anions in an aqueous phase makes it possible to limit the growth of particles in the nanometer range and prevents the formation of aggregates. It is believed that the stabilization of nanoparticles of SHMP arises both due to the electrostatic repulsion of negatively charged phosphate groups, and due to steric effects [9]. It is possible to limit growth due to the chemical interaction of phosphate ions with iron cations in addition.

The advantages of this method include its simplicity and the possibility of obtaining nanoparticles of a certain size by regulating the concentration of the stabilizer.

The main condition for the production of LangmuirBlodgett films (LBF) is the presence of an insoluble monosolayer on the surface of a liquid (usually water) subphase. The thickness of the coating can be accurately determined by transferring a certain number of monolayers to a solid substrate, the LBF composition can be changed from layer to layer, thus obtaining films with molecularly specified properties in addition. In Langmuir-Blodgett technology use a modification of particles by surfactants to form monolayers of nanoparticles on the surface of water for the purpose of their further application. One simple method of surface modification is the extraction of nanoparticles in an organic solvent containing a surfactant, for example cetyltrimethylammonium bromide (CTAB). Anionic surfactants increase the degree of extraction due to electrostatic interaction with positively charged nanoparticles in aqueous solution. A similar method is used for the production of Langmuir-Blodgett films based on polyoxymetallates and cationic surfactants [10].

The layer by layer assembly of multilayers containing particles of Prussian blue can be carried out with the help of oppositely charged polyelectrolytes or lipids [11]. Charged colloidal particles of Prussian blue in the aqueous subphase are transferred to a solid substrate by electrostatic interaction with oppositely charged lipid or polymer molecules at the phase separation interface in this method. However, such a method can not be used for self-assembled assembling of other metal hexacyanoferrates since these compounds do not form a stable colloidal solution even at very low concentration of reacting substances $[12,13]$. Overcoming this disadvantage is possible by introducing stabilizers, which makes it possible to obtain multilayer structures of almost all metal hexacyanoferrates [14].

We obtained Langmuir-Blodgett films based on iron (III) and iron (II) stearates, their structure, surface and magnetic properties were investigated earlier [15]. It is of interest to produce Langmuir-Blodgett films based on monolayers that also contain iron ions, but in the form of nanoparticles in the continuation of the study.

\section{EXPERIMENTAL DETAILS}

The study of the isotherms of compression of monolayers was carried out by the method of continuous compression in an automatic installation consisting of a duralumin paraffined bath in which the test solution was placed, the Langmuir torsion balance, to which a sensitive sensor was connected, and a movable barrier with an automatic movement mechanism at a speed of $5 \mathrm{~cm} / \mathrm{min}=20 \pm 2{ }^{\circ} \mathrm{C}$.

The iron hexacyanoferrate nanoparticles were obtained by the following method. $10 \mathrm{ml}$ of $0.01 \mathrm{M}$ 
aqueous ferric chloride solution were added dropwise with constant stirring to an aqueous mixture containing $15 \mathrm{ml}$ of $0.01 \mathrm{M}$ potassium hexacyanoferrate (II) and $10 \mathrm{ml}$ of a $0.01 \mathrm{M}$ solution of sodium hexametaphosphate (SHMP), which was used as a stabilizer. The nanoparticles of the nickel hexacyanoferrate, cobalt and copper were synthesized in a similar way. $15 \mathrm{ml}$ of a $0.01 \mathrm{M}$ solution of nickel chloride were added to a solution containing $10 \mathrm{ml}$ of $0.01 \mathrm{M}$ potassium ferricyanid and $10 \mathrm{ml}$ of $0.01 \mathrm{M}$ SHMP in the case of synthesis of nanoparticles of the nickel hexacyanoferrate. To prepare nanoparticles of cobalt and copper hexacyanoferrate $1.5 \mathrm{ml}$ of a $0.01 \mathrm{M}$ cobalt (II) nitrate solution or copper sulphate were added to solutions containing $1 \mathrm{ml}$ of $0.01 \mathrm{M}$ potassium ferricyanid and $10 \mathrm{ml}$ of $0.01 \mathrm{M} \mathrm{SHMP}$ (in the case of copper hexacyanoferrate- $0.5 \mathrm{ml} 0.01 \mathrm{M} \mathrm{SHMP}$ ), respectively. Unlike the synthesis of hexacyanoferrates in a volume resulting in the immediate formation of a sparingly soluble precipitate, this procedure allowed the preparation of a transparent opalescent dispersion.

The following reactions were performed for the preparation of salts:

$$
\begin{aligned}
& 3 \mathrm{~K}_{4}\left[\mathrm{Fe}(\mathrm{CN})_{6}\right]+4 \mathrm{FeCl}_{3}= \\
& \mathrm{Fe}_{4}\left[\mathrm{Fe}(\mathrm{CN})_{6}\right]_{3} \downarrow+12 \mathrm{KCl}, \\
& 2 \mathrm{~K}_{3}\left[\mathrm{Fe}(\mathrm{CN})_{6}\right]+3 \mathrm{NiCl}_{2}= \\
& \mathrm{Ni}_{3}\left[\mathrm{Fe}(\mathrm{CN})_{6}\right]_{2} \downarrow+6 \mathrm{KCl}, \\
& 2 \mathrm{~K}_{3}\left[\mathrm{Fe}(\mathrm{CN})_{6}\right]+3 \mathrm{Co}\left(\mathrm{NO}_{3}\right)_{2}= \\
& \mathrm{Co}_{3}\left[\mathrm{Fe}(\mathrm{CN})_{6}\right]_{2} \downarrow+6 \mathrm{KNO}_{3}, \\
& 3 \mathrm{~K}_{4}\left[\mathrm{Fe}(\mathrm{CN})_{6}\right]+3 \mathrm{CuSO}_{4}= \\
& \mathrm{Cu}_{3}\left[\mathrm{Fe}(\mathrm{CN})_{6}\right]_{2} \downarrow+3 \mathrm{~K}_{2} \mathrm{SO}_{4} .
\end{aligned}
$$

Size of nanoparticles were determined by laser diffraction method using Mastersizer 3000. The micrographs of the samples were made using a scanning electron microscope Hitachi S-3400N with an accelerating voltage of $20 \mathrm{kV}$ at an operating distance of $10 \mathrm{~mm}$.

$X$-ray phase analysis was performed using an automatic diffractometer 2DPHaser (Bruker), X-ray tube radiation $-\mathrm{CoK}_{\alpha 1+2}$, mode of operation of the tube $30 \mathrm{kV} / 15 \mathrm{~mA}$, position-sensitive detector. The phase identification was carried out in the software package PDXL- 2 (Rigaku) using a powder diffraction data base Powder Diffraction File (PDF-2, International Center for Diffraction Data, 2011).

Potentiometric measurements were performed by potentiostat Elins $\mathrm{P} 30 \mathrm{I}$ in the voltage range from

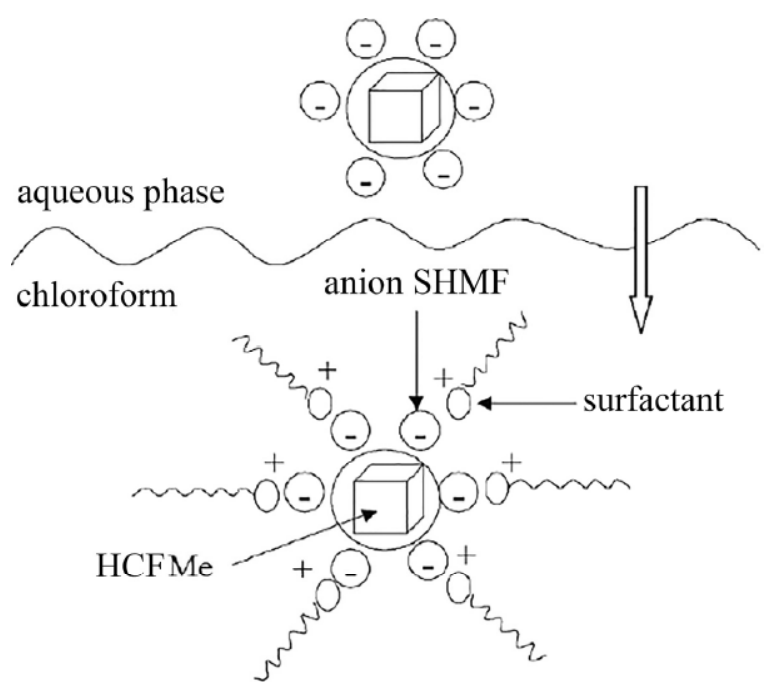

Fig. 1. Schematic representation of the extraction of metal HCF nanoparticles into the organic phase.

0 to $1000 \mathrm{mV}$ at potential scan rates from 10 to 500 $\mathrm{mV} / \mathrm{s}$.

Modification of the particles was carried out according to the following procedure: nanoparticles obtained with the addition of a stabilizer were in aqueous phase. Extraction into the organic phase (chloroform), containing CTAB was carried out for their successful modification by surfactant molecules. Due to the electrostatic interaction between positively charged surfactant ions and the SHMP anions covering the particle, the particles are transferred to the organic phase (Fig. 1).

The particles modified in this way can be transferred to a solid substrate by the Langmuir-Blodgett method. The difference in the compression isotherms may indicate the interaction of nanoparticles with CTAB ions (an indirect sign of the transfer is a change in the color of the organic phase). However, CTAB is a water-soluble surfactant and part of the material remains in the aqueous phase during extraction. Quantitative transfer of nanoparticles is impossible in this case. In addition, the compression isotherms of CTAB monolayers, both before and after modification, contain rectilinear areas, the slope of which indicates a low elasticity of the monolayers, which introduces significant difficulties when they are applied to a solid substrate by the Langmuir-Blodgett method.

Fig. 2 shows the dependence of the surface pressure on the total area, although the dependence of the surface pressure on the area per molecule of surfactant is usually used. This is due to the fact that CTAB is a water-soluble surfactant and uncertainty may arise during the calculation. CTAB was 


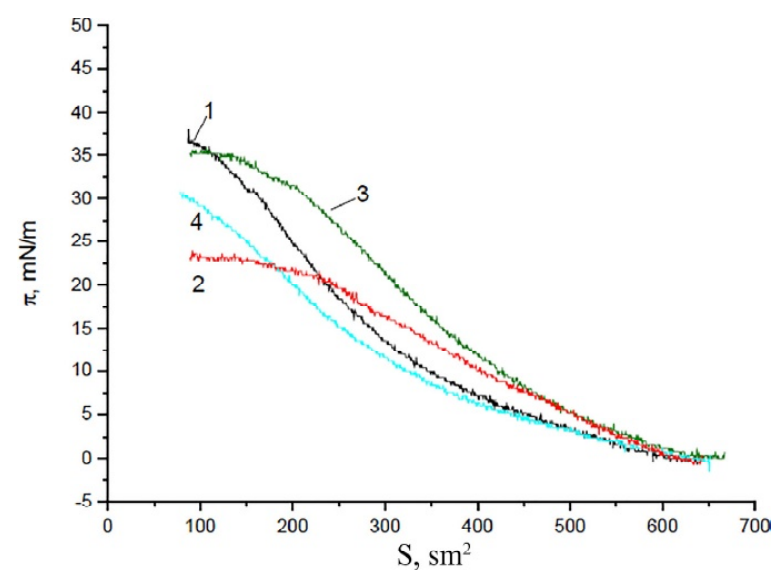

Fig. 2. $\pi$-A isotherms of nanoparticles on water 1 nickel HCF, 2- copper HCF, 3 - cobalt HCF, 4 - iron HCF, modified with CTAB.

replaced with a water-insoluble surfactantoctadecylamine (ODA) to avoid this uncertainty. Being just like CTAB, a cationic surfactant, it allows extraction of nanoparticles into the organic phase, but at the same time forms an insoluble monolayer on the surface of the water subphase.

The research was carried out using the equipment of the resource centers of St. Petersburg State University "Centre for Innovative Technologies of Composite Nanomaterials" and "Centre for GeoEnvironmental Research and Modelling (GEOMODEL)".

\section{RESULTS AND DISCUSSION}

\section{XRD analysis}

The obtained X-ray diffraction patterns (Fig. 3) coincide with that of samples of cobalt HCF, copper HCF, and iron HCF having a cubic syngony with the space

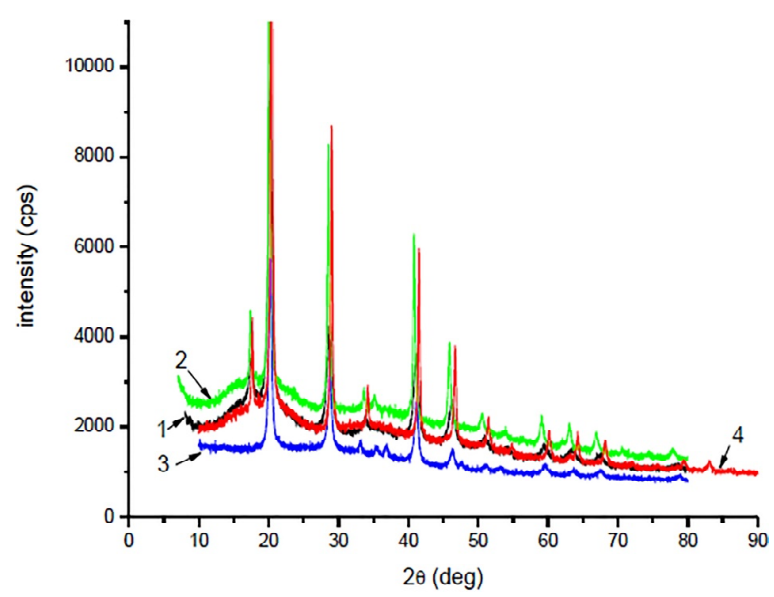

Fig. 3. Types of diffraction patterns of 1 - nickel HCF, 2 - cobalt HCF, 3 - iron HCF and 4 - copper HCF. group Fm3m and a nickel HCF sample having a cubic syngony with the space group F43m.

Determination of particle size by laser diffraction

The results of particle size measurement by laser diffraction (Fig. 4) showed that for the iron HCF sample (Fig. 4a), half of the total number of particles is occupied by objects smaller than $15 \mathrm{~nm}$, for nickel HCF nanoparticles (Fig. 4b) and cobalt (Fig. 4c) this value is less than $16 \mathrm{~nm}$. Larger nanoparticles were obtained for copper HCF (Fig. 4d) (half of the total number of particles occupied by objects smaller than $42 \mathrm{~nm}$ ). The analysis revealed that the dispersions obtained had one fraction with the sizes indicated above, as well as their resistance to aggregation for a long time. In particular, the particle size of the iron HCF $\mathrm{n}$ in the aqueous and in the organic phases remained unchanged for several months, which indicates a high stability of the particles obtained.

\section{$\pi$-A isotherms}

In Fig. $5 \mathrm{a}$, the compression isotherms of monolayer are given for pure ODA and ODA containing iron HCF nanoparticles. The graph of the dependence of the pressure of the ODA monolayer (Fig. $5 a)$, curve 1) on the area per molecule, has two distinct rectilinear sections. The area value when extrapolating the upper linear portion of the isotherm to zero surface pressure is $0.22 \mathrm{~nm}^{2}$, which is close to the value of the cross-sectional area of the hydrocarbon chain. The collapse pressure is about 55 $\mathrm{mN} / \mathrm{m}$.

The isotherm of the compression of a monolayer of ODA containing iron HCF nanoparticles (Fig. $5 a)$, curve 2), has the form similar to the ODA isotherm. An increase in the area of the monolayer is observed for all values of the surface pressure. Two rectilinear sections are observed, the value of the area per molecule at the zero value of the surface pressure is $0.42 \mathrm{~nm}^{2}$.

Fig. 5 also shows the isotherms of compression of pure ODA and a monolayer containing nanoparticles of nickel HCF, cobalt HCF and copper HCF. For nickel HCF, an increase in the monolayer area is observed for almost all values of the surface pressure (Fig. 5b), curve 2). The value of the area per molecule at zero value of the surface pressure is $0.22 \mathrm{~nm}^{2}$ (for a pure ODA this value is $\left.0.20 \mathrm{~nm}^{2}\right)$. The area increases to a value of $20 \mathrm{mN} /$ m (Fig. 5c), curve 2) in the case of cobalt HCF. Extrapolation of the rectilinear section to zero surface pressure gives the value of the area per molecule of $0.35 \mathrm{~nm}^{2}$ in this case. For copper HCF (Figure $5 d$ ), curve 2), an increase in the monolayer area 

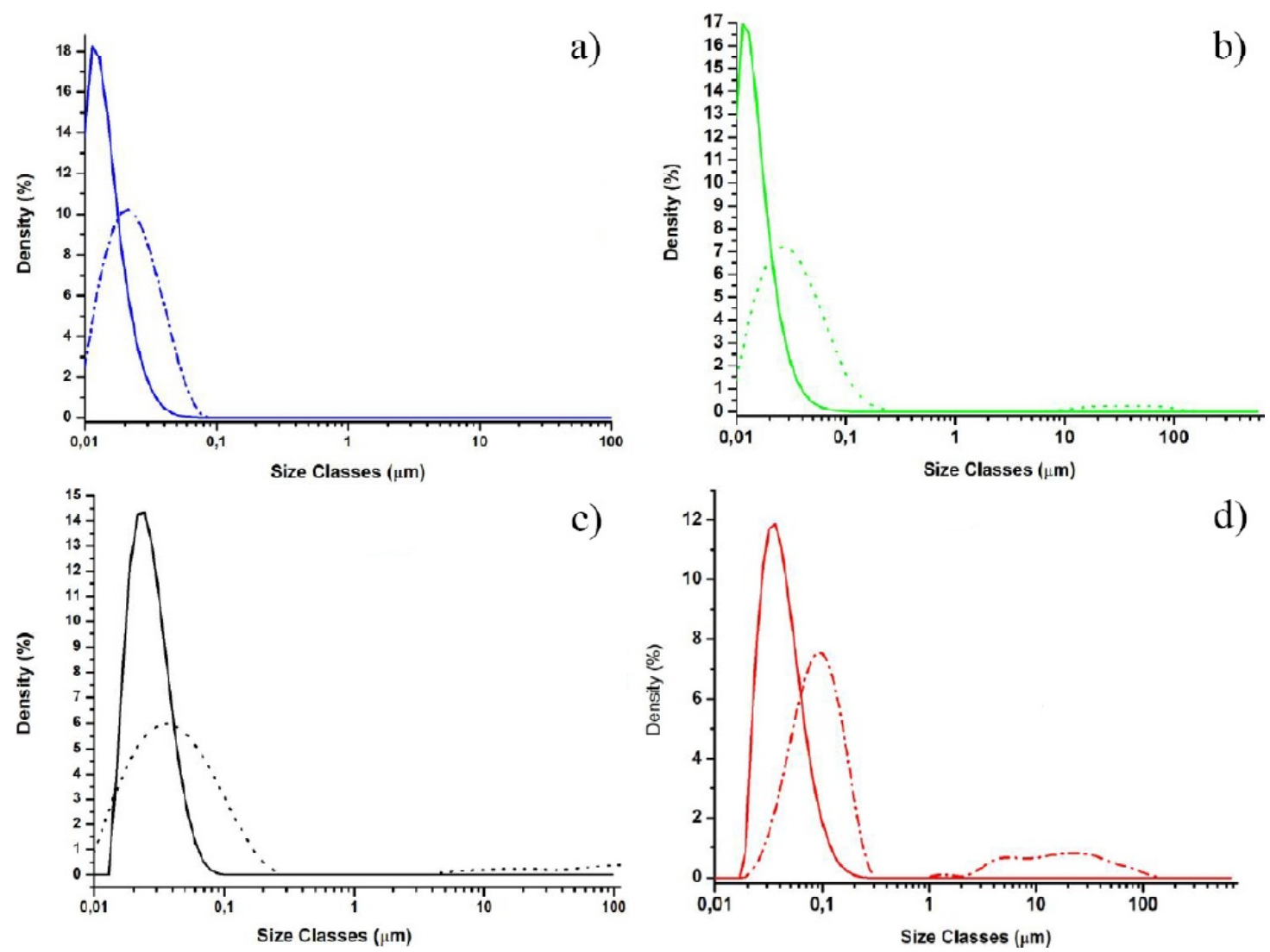

d)

Fig. 4. The volume (dashed line) and numerical (solid line) distributions of particles of iron- a), cobalt - b), nickel - c) and copper - d) hexacyanoferrates.

is observed only at low surface pressures (up to 5 $\mathrm{mN} / \mathrm{m}$ ). The area occupied by the molecule is 0.30 $\mathrm{nm}^{2}$.

It is seen that the course of the dependence for copper HCF particles is very different from all other compression isotherms when comparing the effect of particles of different metals on the surface properties of ODA. There is a decrease in the area per molecule for this system. There is a decrease in the elasticity of the monolayer also. This can be explained by the fact that copper HCF nanoparticles are the largest, their incorporation into a monolayer exerts a disintegrating effect on it and can lead to the escape of some of the molecules from the monolayer.

A change in the form of the compression isotherms indicates a successful modification of the ODA monolayers by particles of metals HCF. The presence of a rectilinear section on compression isotherms for all ODA monolayers modified by HCFs of various metals allows us to conclude that monolayers can be transferred onto a solid substrate.

There is an extraction step that does not allow the quantitative transfer of the metal HCF nanoparticles from the aqueous subphase to the organic subphase in the two above-mentioned methods for the preparation of films. Some of them may remain in the aqueous phase at this stage. We have developed a method to exclude the extraction stage which is as follows. An aqueous solution containing metal HCF nanoparticles is applied dropwise a solution of ODA in chloroform. After evaporation of the solvent on the surface of the water subphase, a monolayer of ODA molecules is formed, with which nanoparticles begin to interact. In this case, an electrostatic interaction appears between the anions of SHMP and the cations of the protonated ODA form. Thus, metal HCF nanoparticles are built into the structure of a surfactant monolayer and can later be transferred to a solid substrate by the LB method. It was previously shown that the obtained hydrosols of the particles of HCF are stable in time, so the sedimentation process can be neglected. As an example, Fig. 6 shows cobalt HCF.

\section{Scanning electron microscopy (SEM)}

In order to obtain information on the structure and size of nanoparticles, as well as on the characteristics of transferred monolayers containing metal HCF nanoparticles, studies were carried out using a scanning electron microscope. The micrographs of freshly prepared samples of metal HCF 

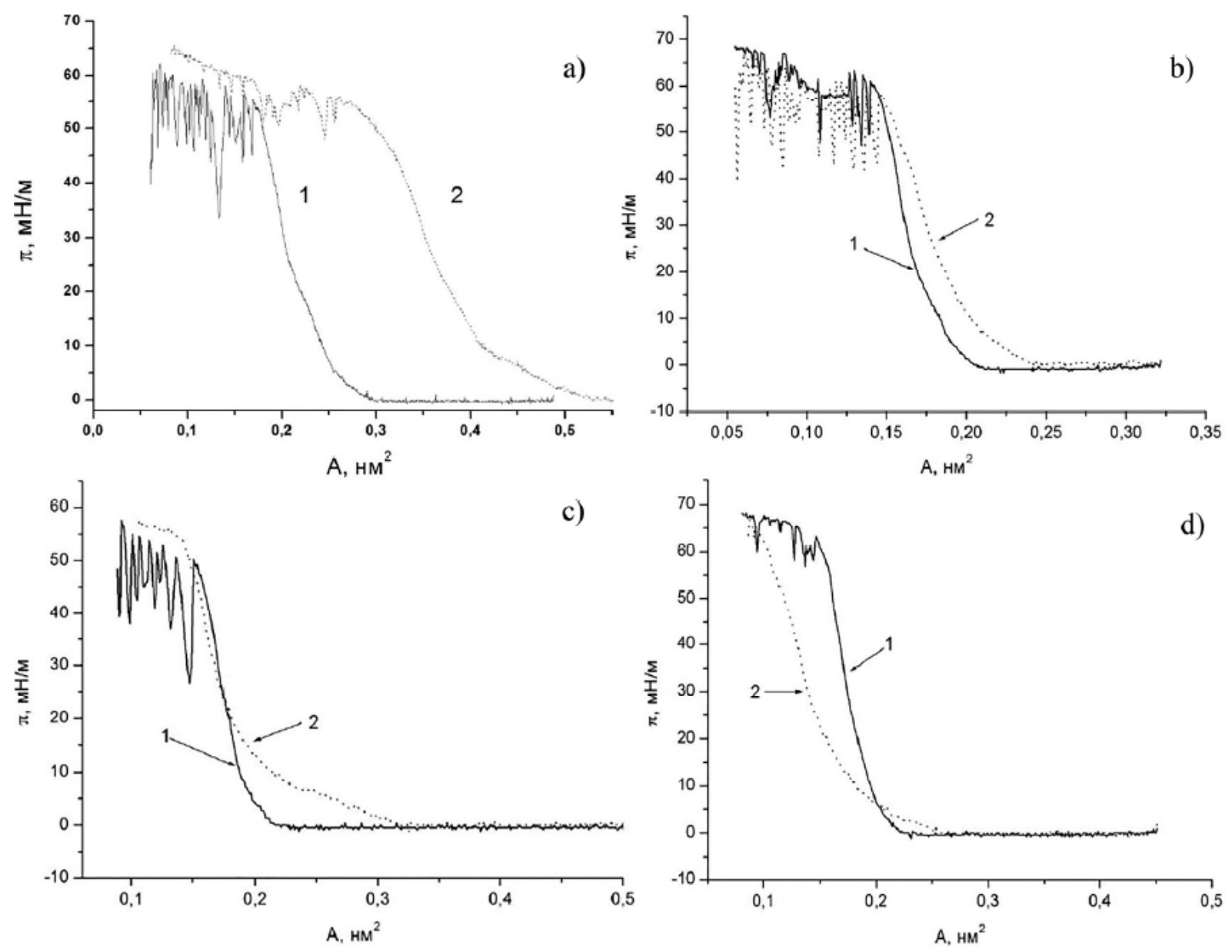

Fig. 5. The compression isotherms of the ODA monolayer on water (1) and the ODA monolayer containing iron - a), nickel - b), cobalt - c) and copper - d) HCF nanoparticles (2).

nanoparticles before extraction are demonstrated in Figs. 7a-7c. The analysis showed that for the dried samples of nickel and cobalt hexacyanoferrates (Figs. 7a-7c), both individual particles and their aggregates are observed. In the image of nanoparticles of nickel HCF on a scale of $50 \mu \mathrm{m}$ (Fig. 7a), large, ordered self-organized structures are seen. It is revealed that these aggregates are formed from filamentary structures (Fig. 7b), which in turn consist of particles (Fig. 7c) the size of which coincides with the size determined by the laser diffraction measurement method with an increase of five times. In the image of the cobalt $\mathrm{HCF}$ (Fig. 7d) aggregates of particles similar in size are observed, but this degree of ordering, as in the case of nickel HCF, is not observed. According to SEM micrographs, copper and iron HCFs are more likely to aggregate than cobalt and nickel HCFs and form crystal-like structures with a developed surface (Figs. $7 \mathrm{e}$ and $7 \mathrm{f}$ ) when the sample is dried. The iron HCF does not form ordered monodisperse particle structures unlike the cobalt and nickel HCFs.
Figs. $8 \mathrm{a}$ and $8 \mathrm{~b}$ show the photomicrographs of ODA monolayers containing iron and cobalt HCF nanoparticles transferred to a solid substrate. It follows from the analysis of images that in the case of iron HCF (Fig. 8a) and in the case of cobalt HCF

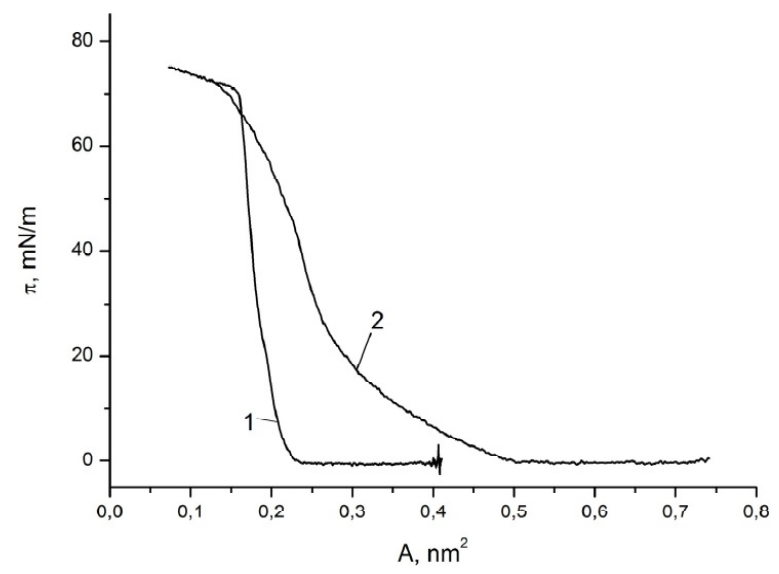

Fig. 6. The compression isotherms of the ODA monolayer on water (1) and the ODA monolayer containing nanoparticles of the nickel $\mathrm{HCF}(2)$. 


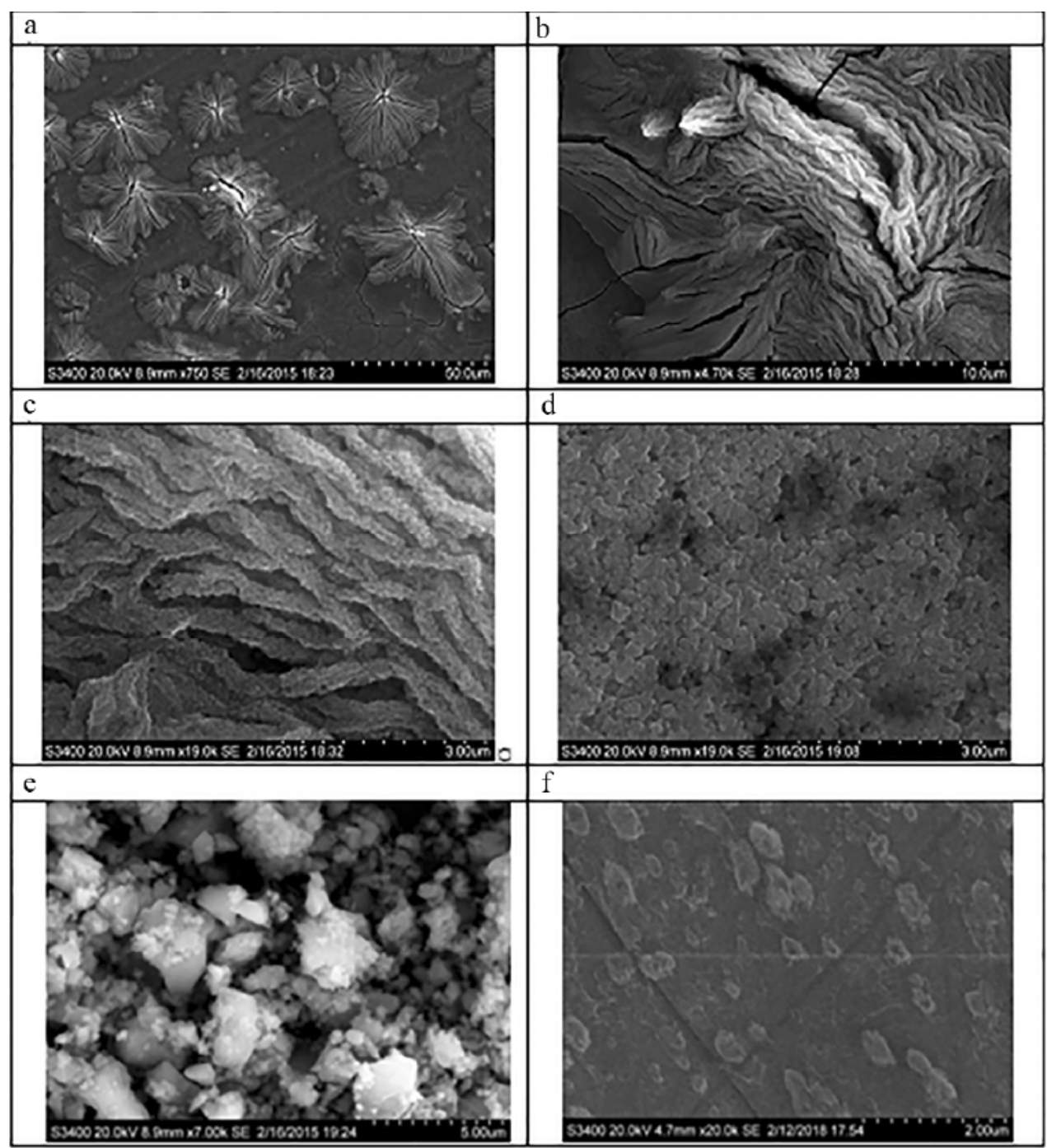

Fig. 7. SEM images of nanoparticles: $a, b, c$ - nickel HCF; $d$ - cobalt HCF, e-copper HCF, $f$-iron HCF.
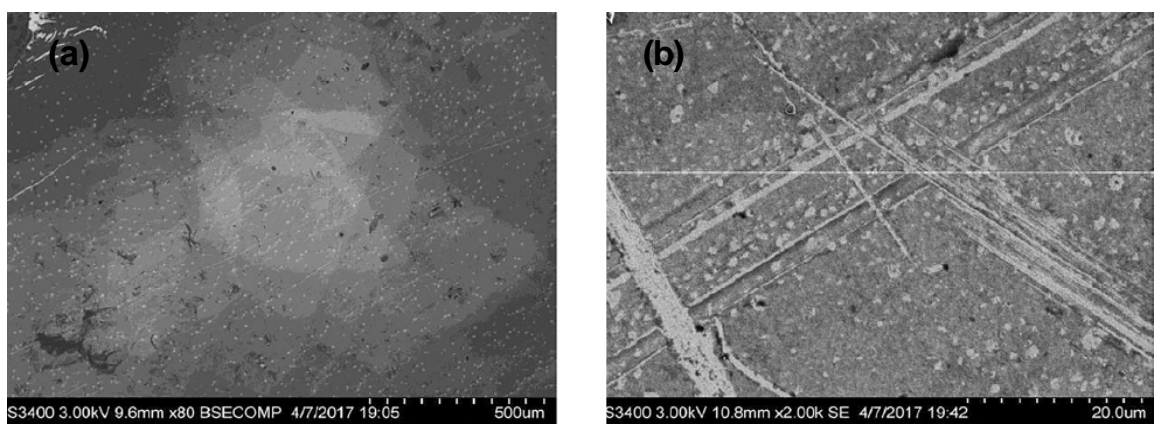

Fig. 8. SEM images of transferred ODA monolayers containing nanoparticles: $a$ - iron HCF, $b$ - cobalt HCF.

(Fig. 8b) aggregates of particles are uniformly distributed in the structure of the film.

\section{Cyclic voltammetry}

ITO substrates with transferred ODA layers containing iron, nickel, cobalt and copper HCFs nanoparticles were investigated by cyclic voltammetry (CVA). The results of CVA confirmed the fact of the transfer of monolayers. From the ob- tained voltammograms it follows that these systems differ from standard electrode systems based on metals HCF (Fig. 9). The peak positions correspond to the transition $\left[\mathrm{Fe}^{\prime \prime}(\mathrm{CN})_{6}\right]^{4-\leftrightarrow} \leftrightarrow\left[\mathrm{Fe}^{\prime \prime \prime}(\mathrm{CN})_{6}\right]^{3}$. The high currents of this oxidation-reduction transition, as well as their proximity to the potential axis, indicate the regularity of the resulting structure [16]. The presence of relatively large capacitive currents indicates 

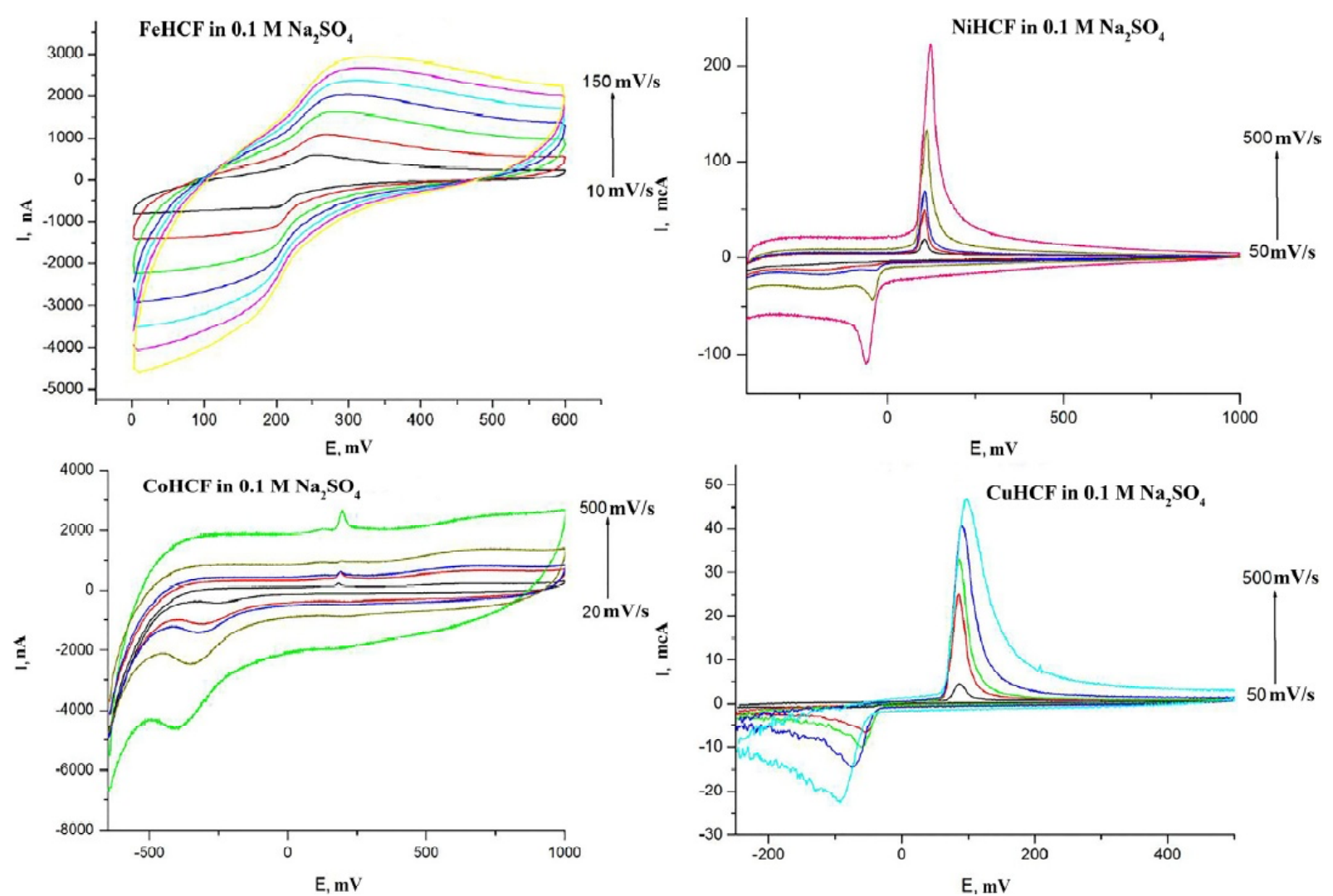

Fig. 9. Cyclic voltammograms of the substrate after the transfer of monolayers of nanoparticles of iron, nickel, cobalt and copper hexacyanoferrate.

the charging of a double layer formed from regular multilayers of surfactants containing $\mathrm{HCF}$ nanoparticles.

\section{CONCLUSIONS}

A simple procedure for the synthesis of iron, nickel, cobalt, copper HCF nanoparticles stabilized by SHMP was developed, consisting in adding to the solution containing potassium ferricyanide and sodium hexametaphosphate, a solution of the salt of the corresponding metal. The average size of the synthesized nanoparticles was determined by the laser diffraction method: iron HCF - $15 \mathrm{~nm}, 16 \mathrm{~nm}$ for nickel and cobalt HCF, copper HCF - $42 \mathrm{~nm}$. With the help of SEM and compression isotherms, a successful modification of the iron, nickel, cobalt and copper HCF nanoparticles by ODA monolayers was proved. The optimal conditions for the transfer of monolayers of iron, nickel, cobalt and copper nanoparticles modified by ODA nanoparticles, to a solid substrate are chosen on the basis of the analysis of pressure isotherms. The technique of transferring monolayers of iron, copper, cobalt and nickel HCF nanoparticles containing ODA to a solid substrate has been worked out. Electrode systems containing from 30 to 40 monolayers of nanoparticles of metal HCF modified by ODA were formed based on the developed procedure for obtaining multimolecular structures. The CVA method confirmed the course of the electrode reaction in the resulting structures corresponding to the transition $\left[\mathrm{Fe}^{\prime \prime}(\mathrm{CN})_{6}\right]^{4-} \leftrightarrow\left[\mathrm{Fe}^{\prime \prime \prime}(\mathrm{CN})_{6}\right]^{3-}$.

\section{REFERENCES}

[1] M. Fitta, H. Prima-Garcia, P. Czaja, T. Korzeniak, M. Krupiński, M. Wojtyniak and M. Bałanda // RSC Adv. 7 (2017) 1382.

[2] N.S. Ivanov, Y. V. Kondrat'ev, A.I. Yanklovich, N.G. Sukhodolov and A.N. Zhukov // Colloid J. 77 (2015) 154.

[3] M. K. Carpenter and R. S. Conell // J. Electrochem. Soc. 137 (1990) 2464.

[4] E.A.R. Duek, M.-A. De Paoli and M. Mastragostino // Adv. Mater. 4 (1992) 287.

[5] N.A. Sitnikova, M.A. Komkova, I. V. Khomyakova, E.E. Karyakina and A.A. Karyakin // Anal. Chem. 86 (2014) 4131.

[6] T. Gupta, A. Kim, S. Phadke, S. Biswas, T. Luong, B.J. Hertzberg, M. Chamoun, K. Evans-Lutterodt and D.A. Steingart // J. Power Sources 305 (2016) 22. 
[7] P.C. Pandey and D. Panday // J. Electroanal. Chem. 763 (2016) 63.

[8] N.S. Ivanov, V.D. Khripun, M.A. Trofimov, N.G. Sukhodolov and A.A. Pendin // Rev. Adv. Mater. Sci. 39 (2014) 34

[9] N. Bagkar, C.A. Betty, P.A. Hassan, K. Kahali, J.R. Bellare and J.V. Yakhmi, // Thin Solid Films 497 (2006) 259.

[10] D.G. Kurth, P. Lehmann, D. Volkmer, A. Müller and D. Schwahn // J. Chem. Soc. Dalt. Trans. (2000) 3989.

[11] A. Jaiswal, J. Colins, B. Agricole, P. Delhaes and S. Ravaine // J. Colloid Interface Sci. 261 (2003) 330.
[12] S. Choudhury, N. Bagkar, G.K. Dey, H. Subramanian and J. V. Yakhmi // Langmuir 18 (2002) 7409.

[13] S. Choudhury, G.K. Dey and J. V. Yakhmi // J. Cryst. Growth 258 (2003) 197.

[14] N. Bagkar, R. Ganguly, S. Choudhury, P.A. Hassan, S. Sawant and J. V. Yakhmi // J. Mater. Chem. 14 (2004) 1430.

[15] N.P. Bobrysheva, N.S. Ivanov, A.A. Selyutin, A.I. Janklovich and N.G. Sukhodolov // Rev. Adv. Mater. Sci. 37 (2014) 48.

[16] A.A. Karyakin // Electroanalysis 13 (2001) 813. 